\title{
WaterGram: A citizen-centric platform to monitor water quality
}

\author{
Iván S. Razo-Zapata*, Giacomo Piovan ${ }^{\dagger}$, and Christian Penny ${ }^{\ddagger}$ \\ ${ }^{*}$ Luxembourg Institute of Science and Technology (LIST) \\ IT for Innovative Services (ITIS) Department \\ 5, avenue des Hauts-Fourneaux. L-4362 Esch/Alzette, GD Luxembourg \\ Email: ivan.razo-zapata@list.lu \\ $\dagger$ Socialmatter \\ Luxembourg, GD Luxembourg \\ Email: giacomo@ socialmatter.eu \\ ${ }^{\ddagger}$ Luxembourg Institute of Science and Technology (LIST) \\ Environmental Research and Innovation (ERIN) Department \\ 41, rue du Brill. L-4422 Belvaux, GD Luxembourg \\ Email: christian.penny@list.lu
}

\begin{abstract}
WaterGram will empower citizens (e.g. students, scientists, designers) and public authorities to make knowledge on water quality more accessible. Even though water is an important asset within our urban environment - from our water tap to the river flowing under a bridge, from the rainwater to the greywater pouring in the canals - citizens are usually detached from the hidden dynamics of water nature. To address this issue, we plan to provide a digital platform that will allow citizens not only to share and monitor information on water quality at anytime but also potentially engage and better understand challenges regarding water management.
\end{abstract}

\section{THE DIGITAL PLATFORM}

Watergram will be a public space in which different stakeholders collaborate to improve water quality monitoring by uploading, sharing and analyzing water measurements. As depicted in Figure 1, the Watergram platform is actually a citizen science initiative that will allow anyone connected to the platform to better understand issues regarding water management. For instance, by collecting water samples at different locations, users can upload that information to Watergram and perform basic analyses such as tracking $\mathrm{pH}$ levels for different locations.

\section{GEO-COVERAGE}

Watergram will be initially launched in the Grand Duchy of Luxembourg where there is a current need to increase capabilities for monitoring water quality. The platform, however, can be potentially replicated in other locations.

\section{MAIN STAKEHOLDERS}

Watergram will be open to public and private participants. On the public side, we foresee the participation of students that will be engaged to use Watergram as well as to build up their own tools to sample water. Moreover, Luxemburgish organisations such as Administration de la Gestion de L'eau and LIST. On the private side, small and medium enterprises

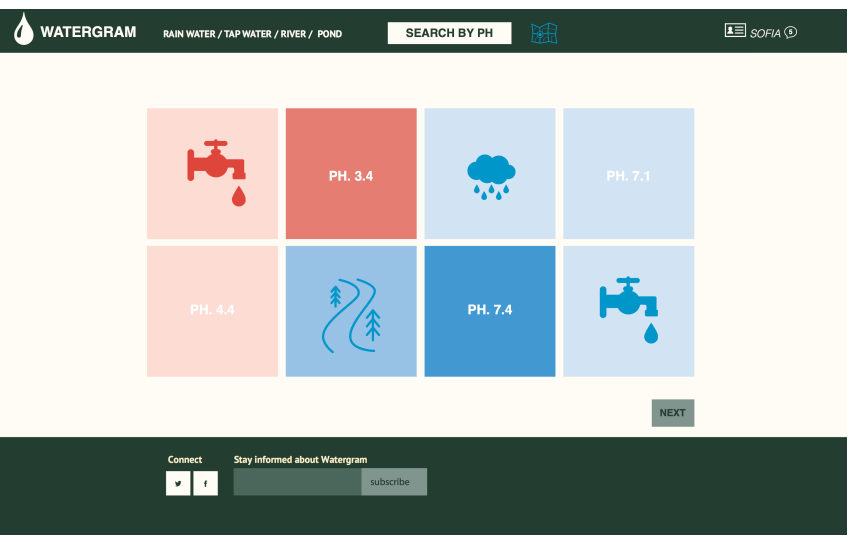

Fig. 1. GUI of the Watergram platform.

may also share data and/or promote the use tools to analyse water samples.

\section{TOOLS FOR SAMPLING AND ANALYSES}

As part of the launch of Watergram, we will also develop workshops to build up so-called Do It Yourself (DIY) tools. These tools will allow Watergram users to collect samples and perform analyses on those samples. We see a lot of potential in these democratic tools because they empower young citizens in understanding environmental science in an interactive way. For example, by using cheap material such as plastic bottles, students can build up filters that can provide different kind of information on water quality. For instance, Figure 2 shows some of these DIY tools that were presented during the Dutch Design Week 2015.

\section{EXPECTED BENEFITS}

Watergram's main benefits are threefold. First, Watergram will facilitate the access to data on water quality, which can 


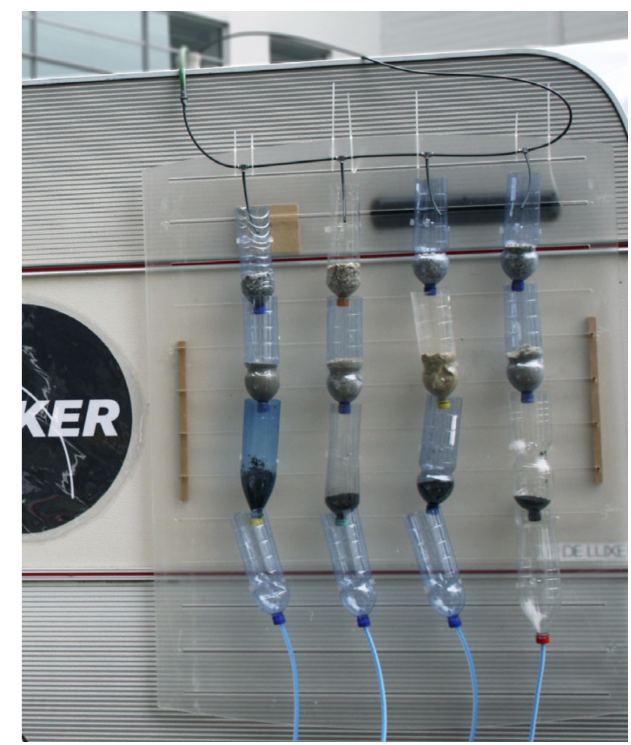

Fig. 2. DIY tools presented during the Dutch Design Week 2015.

also raise awareness among users by comparing data from different users and locations. Second, Watergram will be an open-source platform that can promote collaboration and reuse of data. Finally, Watergram is a citizen science space that can contribute new ideas to the Smart City concept by offering innovative ways to monitor natural resources. 